$\xi=$ 离

\title{
Liposarcoma: experience in a tropical setting
}

\author{
Maurice E Asuquo $^{1 *}$, Martin Nnoli ${ }^{2}$, Victor I C Nwagbara ${ }^{1}$, John Ashindoitiang ${ }^{1}$, \\ Theophilus Ugbem ${ }^{2}$, , Samuel Akpan ${ }^{1}$ \\ ${ }^{I}$ Department of Surgery, University of Calabar, Calabar, Nigeria \\ ${ }^{2}$ Department of Pathology, University of Calabar, Calabar, Nigeria \\ *Corresponding author E-mail: mauefas@yahoo.com
}

\begin{abstract}
Background: Virchow in 1860 described liposarcoma which arise from fat cells. It is a common soft tissue sarcoma found commonly in the limbs and retroperitoneum.

Objectives: This study is an attempt to highlight the biographic indices, pattern, and challenges of management and outcomes of liposarcoma in a tropical setting and proffer solutions for improved outcome.

Methods: Patients who presented to the University of Calabar Teaching Hospital from 2000-2014 with histologic diagnosis of liposarcoma were studied. This was compared with total soft tissue sarcoma and total malignancy seen during the same study period.

Results: The 17 patients seen in the study period comprised 10 males and 7 females $(\mathrm{M}: \mathrm{F}=1.4: 1)$ and their ages ranged from $37-70$ years (mean 51 years). They accounted for $21.8 \%$ of soft tissue sarcoma and $0.9 \%$ of total malignancy. The commonest site involved was the lower limb 8(47\%), while the abdomen ranked second 5(29\%) with the retroperitoneum the commonest abdominal location. Pleomorphic liposarcoma was the commonest $8(47 \%)$ histologic type followed by myxoid $7(41 \%)$. The outcomes were generally poor with one hospital mortality. Sociocultural beliefs, ignorance and poverty were issues highlighted.

Conclusions: Early presentation and diagnosis and treatment is advocated for improved outcome. Establishment of support groups in oncology will further provide health education services and compliment financing of treatment in view of the enormous cost of services and drugs.
\end{abstract}

Keywords: Liposarcoma; Tropical setting.

\section{Introduction}

Liposarcoma (LPS) arise from fat cells; this malignancy was first described by Virchow in 1860, (Schwartz 2015). In adults, it is one of the most common soft tissue sarcoma (STS) with lesions usually found in the limbs and retroperitoneum (RP), (Turkoglu et al. 2014, Mentel \& Fletcher 1995). It is a rare tumour in children, commonly affecting adults between $40-60$ years with a slight male preponderance, (Schwartz 2015, Turkoglu et al. 2014, Weiss 1996). Overall, it accounts for less than $20 \%$ of STS, commonly present as painless slow growing cutaneous mass; however, some may grow rapidly resulting in ulceration, (Schwartz 2015). Lesions located in the RP tend to grow to a large size in the abdomen prior to presentation with symptoms and signs, (Matthyssens et al. 2015). The World Health Organization (WHO) classify LPS into 5 sub types, 1) Well differentiated, 2) dedifferentiated, 3) myxoid, 4) round cell, 5) pleomorphic, (Schwartz 2015, Turkoglu et al. 2014). Surgery remains the main stay of treatment, in addition to the histologic type constitutes the most important prognostic indices, (Mentzel \& Fletcher 1995, Hoffman et al. 2011). We present this study in an attempt to highlight the biographic indices, pattern, and challenges of management and outcomes of LPS in a tropical setting and proffer solutions for improved outcome.

\section{Patients and methods}

Patients who consulted the University of Calabar Teaching Hospital (UCTH), Calabar from January 2000 to December 2014 with histologic diagnosis of LPS were evaluated. Indices obtained were biographic data, clinical presentation, treatment and outcomes. This was compared with total STS and total malignancies seen during the same study period.

\section{Results}

A total of 17 patients presented to the UCTH in 15 years (20002014). Their ages ranged from $37-70$ years (mean 51 years). They were 10 males and 7 females, $\mathrm{M}: \mathrm{F}=1.4: 1$, Table 1 .

\begin{tabular}{lccc}
\multicolumn{4}{c}{ Table 1: Age/ Sex Distribution } \\
\hline Age Range (Yrs) & Sex & Total (\%) \\
& M & F & \\
\hline $1-10$ & & & \\
$11-20$ & & & \\
$21-30$ & 5 & 1 & $6(35.3)$ \\
$31-40$ & 2 & 2 & $4(23.5)$ \\
$41-50$ & 1 & 2 & $3(17.7)$ \\
$51-60$ & 2 & 2 & $4(23.5)$ \\
$61-70$ & 10 & 7 & $17(100)$ \\
Total & &
\end{tabular}


$\mathrm{M}: \mathrm{F}=1.4: 1$

The total number of malignancies seen during the same study period was 1988 and the 17 patients with LPS accounted for $0.9 \%$ of total malignancies, Table 2 .

Table 2: Soft Tissue Sarcoma $(2000-20014)$

\begin{tabular}{ll}
\hline & No $(\%)$ \\
\hline Rhabdomyosarcoma & $27(34.6)$ \\
Liposarcoma & $17(21.8)$ \\
Malignant Fibrous Histiocytoma & $7(9.0)$ \\
Fibrosarcoma & $7(9.0)$ \\
Synovial Sarcoma & $7(9.0)$ \\
Neurofibrosarcoma & $5(6.4)$ \\
Leiomyosarcoma & $3(3.8)$ \\
Malignant Peripheral & \\
Nerve Sheath Tumour (MPNST) & $3(3.8)$ \\
Chondrosarcoma & $2(2.6)$ \\
Total & $78(100 \%)$ \\
\hline
\end{tabular}

Total malignancy $(2000-2014)=1988$

LPS 17 patients, $0.9 \%$ of total malignancy
Seventy eight patients with STS seen during the study period accounted for $4 \%$ of total malignancy while the 17 patients with LPS accounted for $21.8 \%$ of STS and ranked $2^{\text {nd }}$.

The commonest site involved was the lower limb 8(47\%), Figure 1.

The duration of illness prior to presentation ranged from 6-60 months [mean 16 months (1 year 4 months)]. Late presentation with advanced lesions was a common finding as majority of the patients $14(82.4 \%)$ had consulted other health facilities tradomedical inclusive. All the patients presented with swellings with some ulcerated in cutaneous locations, while others presented as abdominal swellings in retroperitoneal and intraperitoneal locations. All the 5 patients with abdominal lesions had laparotomy, 3 retroperitoneal lesions had incision biopsy on account of advanced lesions while the other 2 intraperitoneal lesions had excision for mesenteric LPS. Out of the 9 limb lesions, 5 were offered excision while 4 had incision biopsy. The other lesions located on the head and chest had incision biopsies but for one who had excision biopsy.

The commonest histological type was pleomorphic 8(47\%), Figure 3.

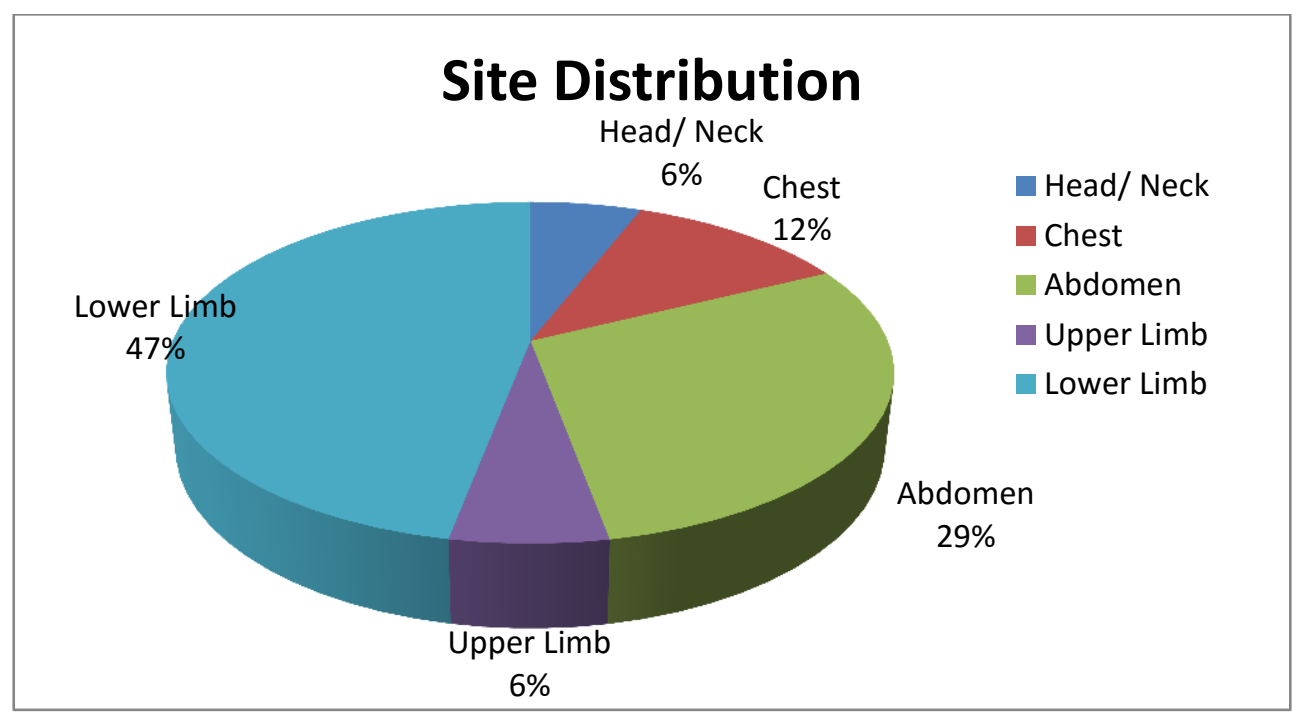

Fig. 1: Site Distribution.

The thigh was the commonest location 4(50\%), [compression of lesion showed lobulations], Figure 2, leg 2(25\%) and foot 2(25\%). The $2^{\text {nd }}$ site in ranking was the abdomen 5(29\%), [Retroperitoneal 3(17.4\%), intraperitoneal $2(11.6 \%)$ ]. Other sites were chest $2(12 \%)$, head and neck 1(6\%) and upper limb 1(6\%).

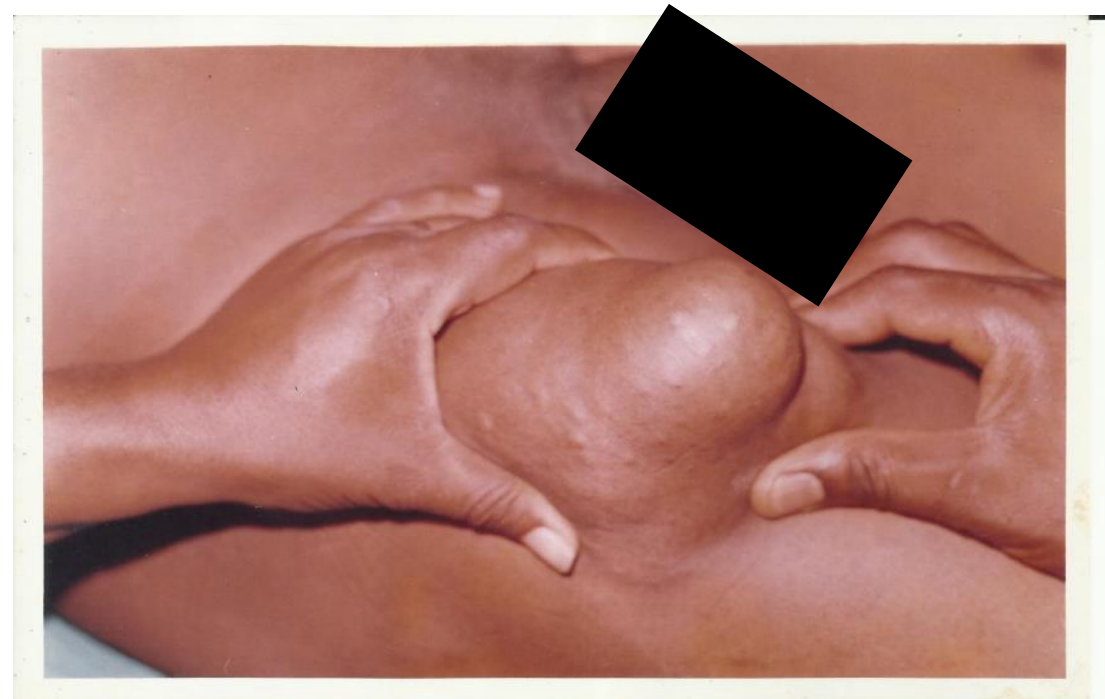

Fig. 2: Clinical Photograph, Liposarcoma (Right Upper Thigh). 


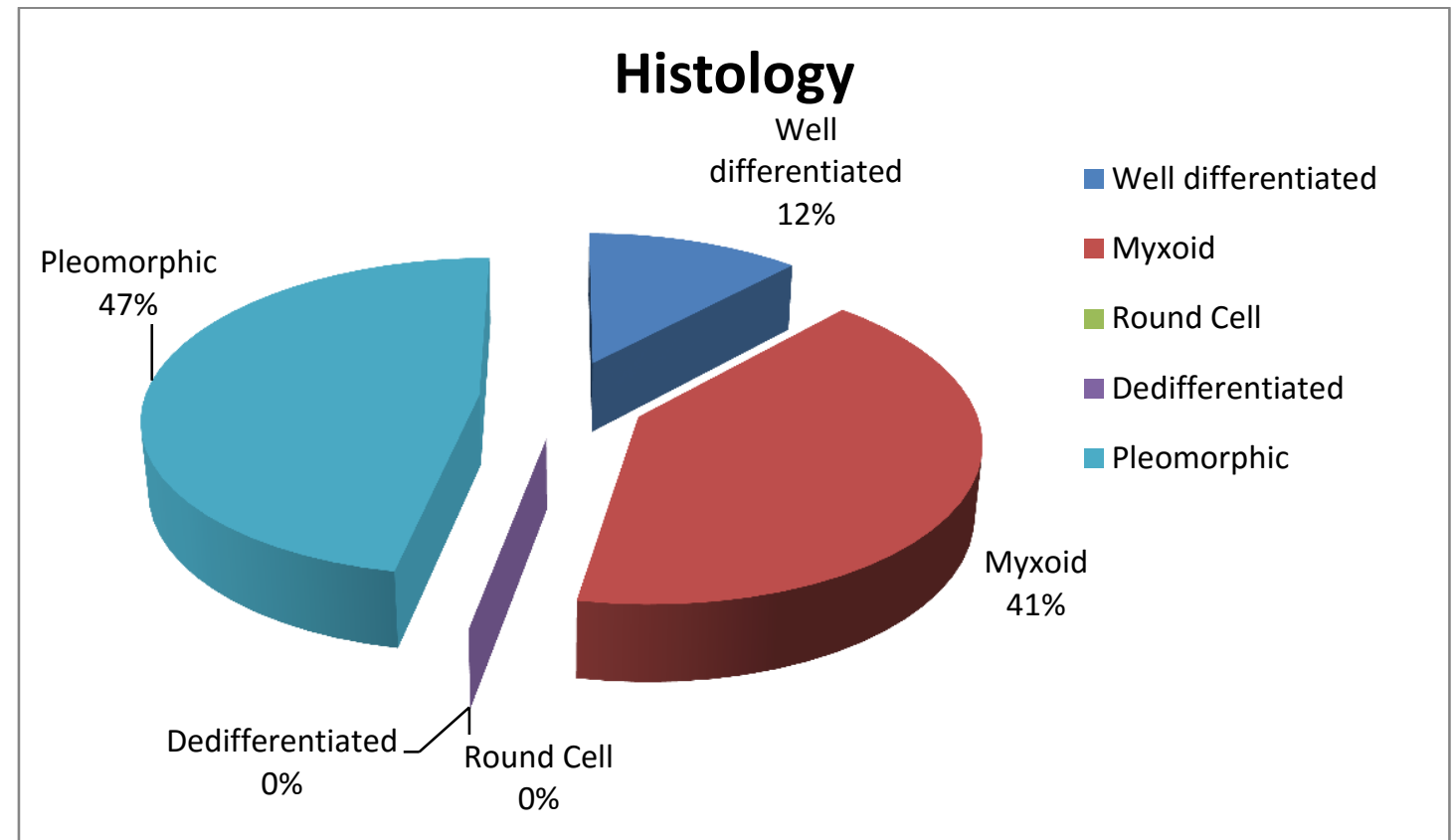

Fig. 3: Histologic Types.

Figure 4 shows tumour composed of malignant mesenchyma. The cells are pleomorphic, round spindle in some areas, some giant cells and lipoblasts cells. There is brisk mitosis; abnormal types of quadripolar mitosis - pleomorphic LPS. Myxoid LPS ranked second $7(41 \%)$,

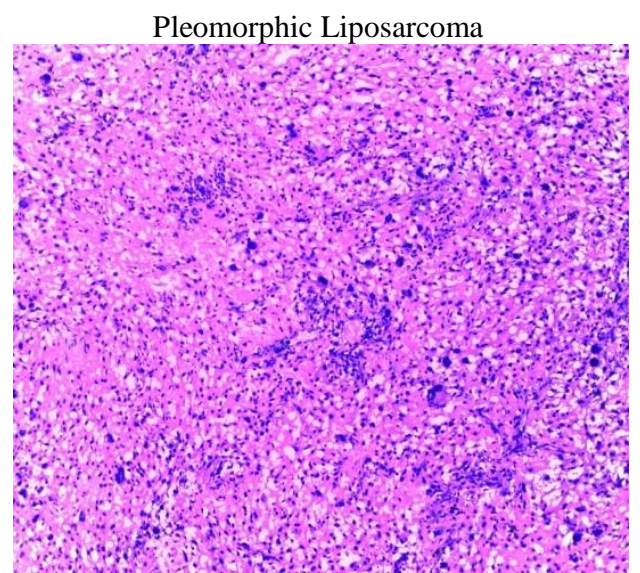

Fig. 4a: (Top) H\&E X 40

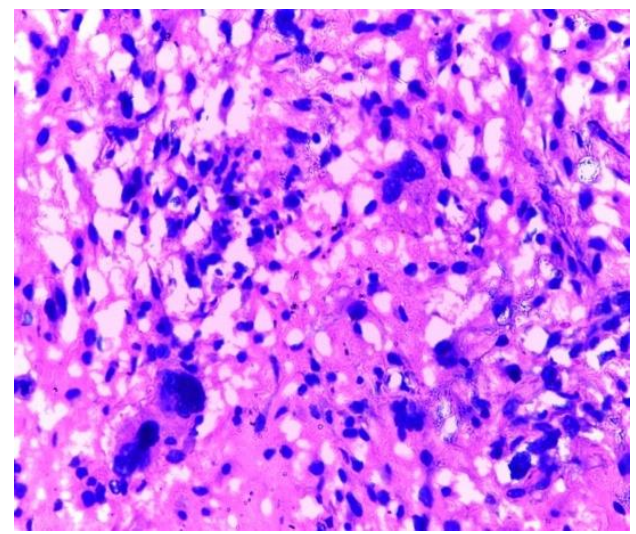

Fig. 4b: (Bottom) - H\&E x 100

Figure 5a\&b. It revealed tumour composed of lobules of myxoid tumour. At low power, Figure 5a, some foci had chicken wire kind of capillaries ramifying tumour tissue. There was adipocytic differentiation with some cells resembling lipoblasts.

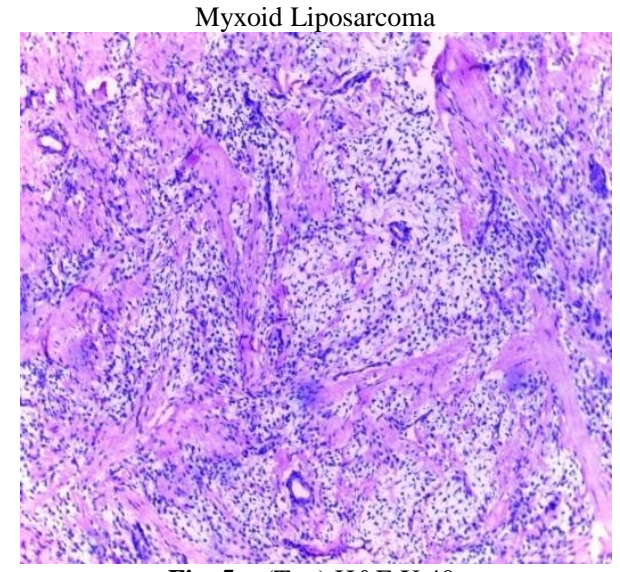

Fig. 5a: (Top) H\&E X 40

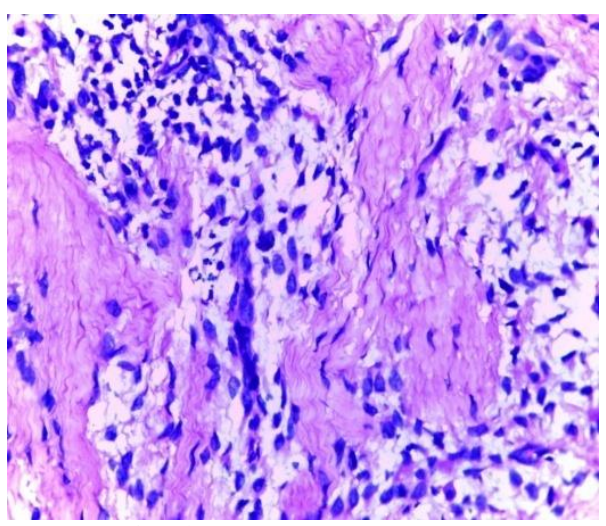

Fig. 5b: (Bottom) - H\&E X 100

No patient was offered pre or postoperative radiotherapy as we did not have this facility. Those referred were lost to follow up. Those that had chemotherapy with doxorubicin had issues purchasing this expensive drug.

The outcomes were generally poor. Two patients with lower limb lesions developed recurrence at 3 and 5 months post excision and were eventually lost to follow up. Follow up of patients ranged from 2-6 months after which most were lost to follow up. Four patients left against medical advice. There was one hospital mortality in a patient with RP LPS. 


\section{Discussion}

Liposarcoma is a common STS accounting for $20 \%$ of malignancies of mesenchymal origin, (Matthyssens et al. 2015, Dei Tos 2000). This is in consonance with our finding of $21.8 \%$, Table 2. Matthyssens et al. 2015 in a similar report showed that LPS accounted $20 \%$ of all STS's while STS was less than $1 \%$ of malignant tumours in adults, (Matthyssens et al. 2015). The latter collaborated with our result of $0.9 \%$. In another study, by Costea et al. 2011, LPS was reported to have accounted for 10-35\% of STS's. Further report on LPS showed it as a common adult STS that ranked $2^{\text {nd }}$ to malignant fibrous histiocytoma (MFH), (Nemanqani et al. 1999). This report is in agreement with our finding of LPS that ranked $2^{\text {nd }}$ but differed from our finding of rhabdomyosarcoma that ranked $1^{\text {st }}(34.6 \%)$, Table 2 .

The mean age in this study was 51 years with no patient recorded in the first 3 decades of life. In keeping with this report, Schwartz 2015 reported a mean age of 50 years, (Schwartz 2015). Liposarcoma in children is reported to be less than 5\% of STS with about 60 children reported in literature. Our report further confirms the rarity of this tumour in the younger age group. There was a slight male preponderance, (Schwartz 2015), Table 1.

It is common to find LPS in the extremities followed by the RP and less frequently in the head and neck in keeping with the results of this study, (Schwartz 2015, Turkoglu et al. 2014, Mentzel $\&$ Fletcher 1995), Figure 1. In the lower limb (the commonest site), the commonest location was the thigh, Figure 2, (Nemanqani et al. 1999). The head and neck is an uncommon site with the oral location very rare, this was the only head and neck location reported in this study. Up to the year 2000 less than 50 cases of oral involvement has been reported, (Schwartz 2015). Transformation of lipoma into LPS is reported to be rare, as a rule well differentiated LPS do not arise from lipoma which lack genetic markers consistent with LPS, (Mentzel \& Fletcher 1995).

This lesion tends to grow in an exophytic fashion be it in the cutaneous, RP or oral locations, presenting as polypoid or dome shaped mass, Figure 2. It is believed to be centred in the dermis in the cutaneous setting with minimal tendency to grow into the sub cutis, (Schwartz 2015, Dei Tos 2000). Tumours in the RP location grow into a voluminous size prior to discovery; this is due to the expandable disposition of the abdomen, (Matthyssens et al. 2015). This was in keeping with our experience as all the patients with RP tumours presented late. The mean duration of illness prior to presentation was 16 months, however, in view of the above stated, patients estimation of clinical duration of illness is usually unreliable, (Schwartz 2015). Late presentation may have impacted adversely on the outcomes, early presentation, early diagnosis, especially with lesions in cutaneous locations is recommended.

Despite WHO classification of LPS into 5 categories, well differentiated, dedifferentiated, myxoid, round cell and pleomorphic, (Schwartz 2015, Turkoglu et al. 2014), myxoid and round cell share morphological and clinical features including similar chromosomal markers, (Dei Tos 2000, Orivieto et al. 2001). Following current data available, the most logical classification of LPS is into 3 distinct groups, 1) well differentiated, 2) myxoid/round cell and 3), pleomorphic, (Schwartz 2015, Dei Tos 2000). Well differentiated and dedifferentiated LPS are reported to account for 40$50 \%$ of adult LPS at variance with our finding of $12 \%$. (Costea et al. 2011). Myxoid and round cell LPS represented $41 \%$ in our study, while Orvieto et al. 2001 in their study reported 30-35\%. Contrary to our finding (Pleomorphic $47 \%$, myxoid $41 \%$, well differentiated $12 \%$ ), Nemanqani D et al. reported myxoid LPS $(50.2 \%)$ as the commonest followed by well differentiated (21.9\%), (Nemanqani et al. 1999). The relative paucity of well differentiated LPS in our setting may have accounted for the poor outcomes in addition of late presentation as histologic type is a known prognostic factor, (Mentzel \& Fletcher 1995), Hoffman et al. 2011).

Surgery is the main stay of treatment with possible cure in nonmetastatic disease, (Matthyssens et al. 2015, Hoffman et al. 2011) Radiation (pre and post) therapy is a valuable adjunct aimed at local control and preoperative treatment offers a good chance recurrence free interval, In management of advanced or metastatic LPS, systemic treatment has an established role in palliation using active agents as doxorubicin, Epirubicin and ifosfamide, (Matthyssens et al. 2015). However, late presentation was prevalent in our setting; this precluded possible curative surgical excision.

In summary, LPS ranked $2^{\text {nd }}(21.8 \%)$ of STS's and accounted for $0.9 \%$ of total malignancies. There was a slight male preponderance with a mean age of 51 years. The commonest site was the lower limb (thigh as the commonest lower limb location), with retroperitoneal site that ranked second. There was a case of oral LPS a rare site of this pathology. The commonest histologic type was pleomorphic followed by myxoid.

\section{Conclusion}

Ignorance, sociocultural beliefs, poverty were significant underlying issues responsible for late presentation which impacted negatively on outcomes. Early presentation, early surgery, provision of affordable adjunct therapeutic facilities are advocated for improved outcome. The use of support groups in oncology to facilitate health education and finance will further improve outcome.

\section{References}

[1] Schwartz RA Liposarcoma 2015. Available at http://emedicine.medscape. Com/article/1102007-overview updated May $28^{\text {th }}$.

[2] Turkoglu MH, Elpek GO, Dogru V, Calis H, Ucar A, Arici C 2014 An unusual case of primary colonic dedifferentiated liposarcoma. Int $J$ of Surg Case Report 5: 8-11. http://dx.doi.org/10.1016/j.ijscr.2013.10.013.

[3] Mentzel T, Fletcher CD 1995. Lipomatous tumours of soft tissues: an update. Virchows Arch 427(4):353-363. http://dx.doi.org/10.1007/BF00199383.

[4] Weiss SW 1996. Lipomatous tumours. Monogr Pathol 38: 207-239.

[5] Matthyssens LE, Creytens D, Ceelen WP 2015. Retroperitoneal liposarcoma: current insights in diagnosis and treatment. Frontiers in Surg 2/Article 4: 1-20

[6] Hoffman A, Lazar AJ, Pollock RE, Lev D 2011. New frontiers in treatment of liposarcoma, a therapeutically resistant malignant cohort. Drug Resist. Updat.14 (1): 52-66. http://dx.doi.org/10.1016/j.drup.2010.11.001.

[7] Dei Tos AP 2000. Liposarcoma new entities and evolving concepts. Ann Diagn Pathol 4(4): 252-266. http://dx.doi.org/10.1053/adpa.2000.8133.

[8] Costea R, Vasiliu E, Zamescu NO, HasounaM, Neagu S, Davila C 2011. Large thigh liposarcoma- Diagnostic and therapeutic features. Journal of Medicine and Life 4(2): 184-188.

[9] Nemanqani D, Mourad WA, Akhtar M, Moreau P, Rostom A, Ezzat A, Amin T 1999. Liposarcoma: A clinicopathological study of 73 cases diagnosed at King Faisal Specialist Hospital and Research Centre. Ann Saudi Med 19(4): 299-303.

[10] Orvieto E, Furlanetto A, Laurino L, Dei Tos AP 2001. Myxoid and round cell liposarcoma: a spectrum of myxoid adipocytic neoplasia. Semin Diagn Pathol 18(4): 267-273. 